\title{
Dentigerous Cyst Associated with Impacted Third Molar
}

\author{
${ }^{1}$ MS Nalini, ${ }^{2}$ Balasubramanya Kumar, ${ }^{3}$ Sunil Shroff
}

\begin{abstract}
A dentigerous cyst is an odontogenic cyst associated with the crown of the impacted or unerupted teeth. Such cyst remains initially completely asymptomatic unless when infected and can be discovered only on routine radiographic examination. Here is a case of dentigerous cyst, present in left mandibular region associated with cortical expansion and facial asymmetry which has been enucleated and the tooth extracted surgically is discussed in present case report.
\end{abstract}

Keywords: Case report, Dentigerous cyst, Ectopic tooth Impacted, Odontogenic cyst.

How to cite this article: Nalini MS, Kumar B, Shroff S. Dentigerous Cyst Associated with Impacted Third Molar. J Health Sci Res 2015;6(1):11-13.

Source of support: Nil

Conflict of interest: None

\section{INTRODUCTION}

The dentigerous cyst is the second most common cyst of the jaws comprising $14-20 \%$ of all jaw cysts, and they are more frequent in males and more common in mandible. ${ }^{1-4}$ By definition, this lesion is attached to the cervix of an impacted tooth and results from proliferation of reduced enamel epithelium after the enamel formation. Dentigerous cysts are usually discovered on routine radiographic examination or when radiographs are taken to determine the reason for failure of a tooth to erupt. They are always radiolucent and usually unilocular, although large lesions occasionally show a scalloping multilocular pattern. ${ }^{3-6}$

The frequency of dentigerous cyst formation has been estimated to constitute 1.44 per 100 unerupted teeth. $^{7}$ Furthermore, the risk for individual teeth to develop dentigerous cyst varies considerably. In case of mandibular

${ }^{1}$ Reader, ${ }^{2}$ Head, ${ }^{3}$ Fellow

${ }^{1}$ Department of Periodontics, Rajarajeswari Dental College and Hospital, Bengaluru, Karnataka, India

${ }^{2}$ Department of Dentistry and Maxillofacial Surgery, St Martha's Hospital, Bengaluru, Karnataka, India

${ }^{3}$ Department of Esthetic Facial Surgery, Bhagwan Mahaveer Jain Hospital, Bengaluru, Karnataka, India

Corresponding Author: MS Nalini, No-005, Sumo Krupa Temple View Apartment, Near Subramanyapura Post Office, Gubbalala, Bengaluru-61, Karnataka, India Phone: 9986396654, e-mail: drnalinims79@gmail.com third molars, the frequency of impaction is roughly the same as that of cyst formation, whereas maxillary third molars have a much higher frequency of impaction than cyst involvement, suggesting that this tooth has a much lower relative risk of developing a dentigerous cyst than its mandibular counterpart. ${ }^{8}$ Similarly, the risk of cyst formation around the crowns of unerupted mandibular first premolars, maxillary incisors, or mandibular second molars is very high, although the frequency of failure of eruption of these teeth is extremely low. ${ }^{9}$

Most dentigerous cysts are asymptomatic but may attain large size with resorption of the roots of teeth till it manifests clinically or radiographically. Treatment of cyst consists of removal of unerupted tooth. Prognosis is excellent and recurrence is rare if completely removed.

\section{CASE REPORT}

A 20 years old female patient reported to our clinic with chief complaint of swelling on the lower left side of her face which was present since 3 months. The patient gave history of pain in that region since 1 month (Fig. 1). Extraoral examination revealed swelling in the left mandibular region near the angle of mandible and extending inferiorly to the lower border of the mandible. The swelling was firm in consistency and tender on palpation. Intraoral examination revealed a swelling extending laterally from distal surface of mandibular left first molar, inferiorly obliterating vestibule and superiorly up till gingival margin. Slight tenderness was noticed on percussion with first and second molar and grade II mobility on left second molar.

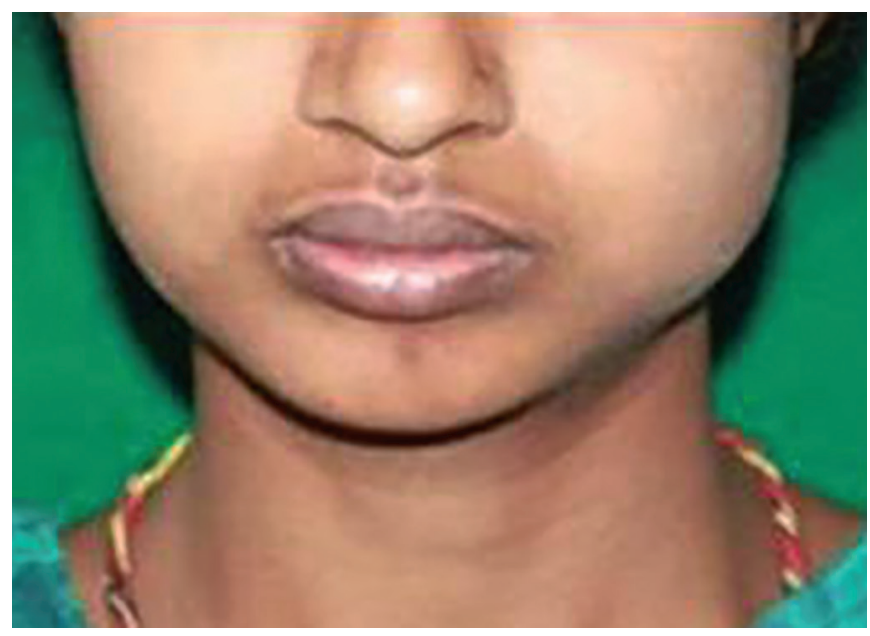

Fig. 1: Preoperative photograph showing extraoral swelling on left mandibular region 


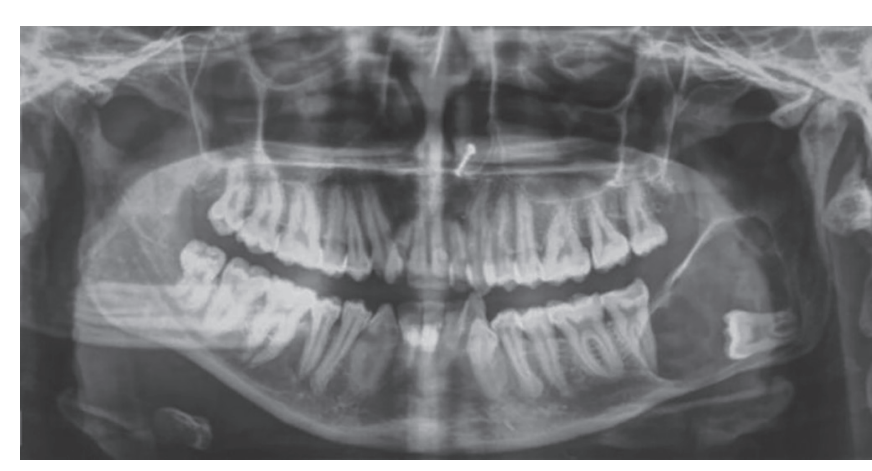

Fig. 2: Radiograph showing radiolucency with sclerotic border including left second molar

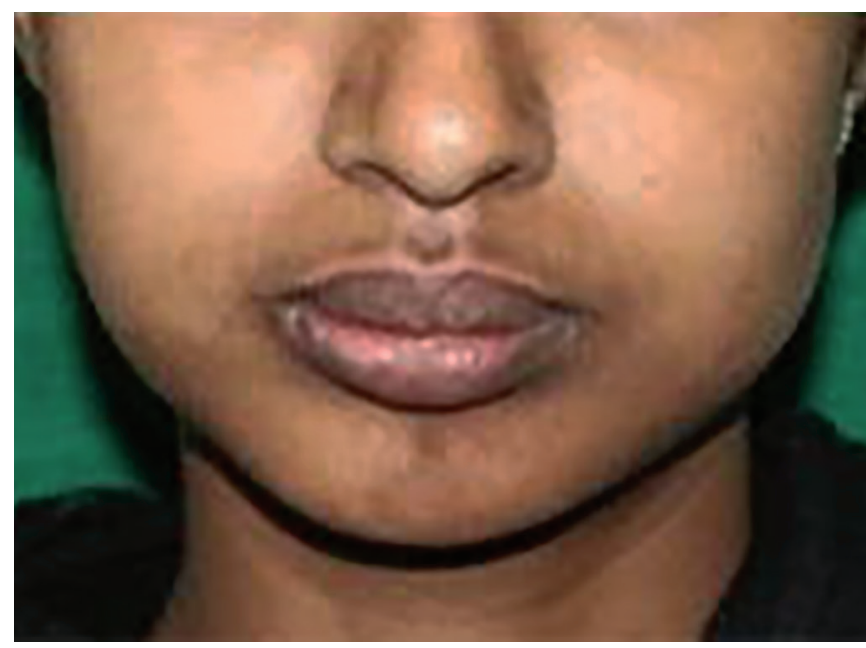

Fig. 3: Postoperative photograph showing absence extraoral swelling

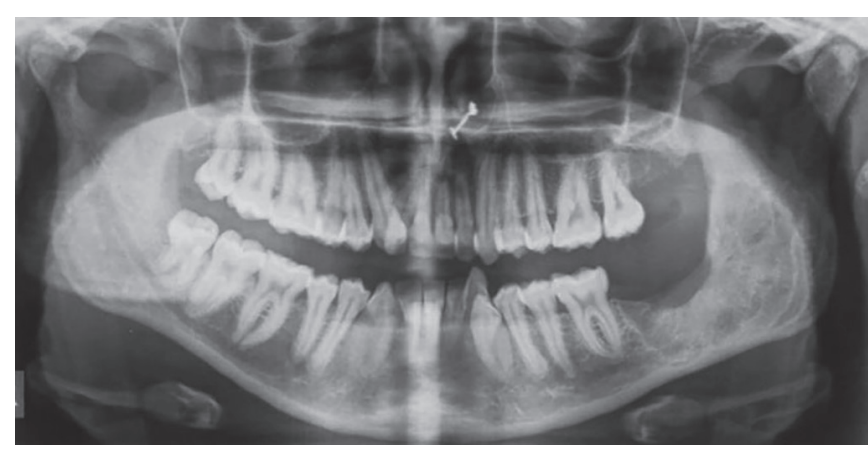

Fig. 4: Radiograph showing healthy bone formation with no recurrence

The panoramic radiographs (Fig. 2) showed radiolucency involving left second molar which was large unilocular, well-circumscribed radiolucent lesion enveloping the unerupted third molar. Based on the aforementioned findings, it was provisionally diagnosed as dentigerous cyst with a differential diagnosis of odontogenic keratocyst or radicular cyst for which enucleation was considered as treatment option. Under local anesthesia, the buccal flap was raised and the cysts enuleated together with associated second and third molar teeth. The mucoperiosteal flap was replaced and sutured. Healing was uneventful, and 1 week after the surgery, the surgical site showed good healing the extraoral swelling was decreased (Fig. 3) 1 month OPG showed no evidence of recurrence of the cysts (Fig. 4).

On the basis of clinical and radiographic findings, the present case was diagnosed as dentigerous cyst of the inflammatory origin involving the unerupted third molar.

\section{DISCUSSION}

A dentigerous cyst is an odontogenic cyst, thought to be of developmental origin-associated with the crown of an unerupted tooth. The cyst cavity is lined by epithelial cells derived from the reduced enamel epithelium of the tooth forming organ. Regarding its pathogenesis, it has been suggested that the pressure exerted by an erupting tooth on the follicle may obstruct venous flow inducing accumulation of exudate between the reduced enamel epithelium and the tooth crown.

In addition to the developmental origin, some authors have suggested that periapical inflammation of nonvital deciduous teeth in proximity to the follicles of unerupted permanent successors may be a factor for triggering this type of cyst formation.

Histologically, a normal dental follicle is lined by enamel epithelium, whereas a dentigerous cyst is lined by nonkeratinized stratified squamous epithelium. Since, the dentigerous cyst develops from follicular epithelium it has more potential for growth, differentiation and degeneration than a radicular cyst. Occasionally, the wall of a dentigerous cyst may give rise to a more ominous mucoepidermoid carcinoma. Due to the tendency for dentigerous cysts to expand rapidly, they may cause pathological fractures of jaw bones. On fine needle aspiration, thin straw colored fluid is seen.

The radiographic appearance is that of a well-demarcated radiolucent lesion attached to the cervical area of an unerupted tooth. Radiographically, a dentigerous cyst should always be differentiated from a normal dental follicle. Dentigerous cysts are the most common cysts with this radiographic appearance. Radiographically, the cyst appears unilocular with well defined margins and often sclerotic borders but sometimes it may be multilocular in appearance and may also have a continuous cystic membrane. Infected cysts show ill-defined margins. The most common location of dentigerous cysts are the mandibular 3rd molars and the maxillary canines, and they rarely involve deciduous teeth and are occasionally associated with odontomes. A dentigerous cyst is often treated by excision of the cyst along with the extraction of the associated tooth. In case of a large cyst marsupialization is done. 


\section{REFERENCES}

1. Assael LA. Surgical management of odontogenic cysts and tumors. In Peterson LJ, Indresano TA, Marciani RD, Roser SM. Principles of Oral and Maxillofacial Surgery. Philadelphia: JB Lippincott, 1992;2:685-688.

2. Neville BW. Odontogenic cysts and tumors. In: Neville BW, Damm DD, Allen CM, Bouquot JE, editors. Oral and maxillofacial pathology. WB Saunders, Philadelphia; 1995. p. 493-496.

3. Regezi JA. Cyst and cyst-like lesions. In: Regezi JA, Sciubba J, Pogrel MA, editors. Atlas of oral and maxillofacial pathology. WB Saunders, Philadelphia; 2000. p. 88.

4. Martínez-Pérez D, Varela-Morales M. Conservative treatment of dentigerous cysts in children: report of four cases. J Oral Maxillofac Surg 2001;59(3):331-334.
5. Dammer R, Niederdellmann H, Dammer P, et al. Conservative or radical treatment of keratocysts: a retrospective view. $\mathrm{Br} \mathrm{J}$ Oral Maxillofac Surg 1997;35(1):46-48.

6. Aguiló L, Gandía JL. Dentigerous cyst of mandibular second premolar in a five-year-old girl, related to a non-vital primary molar removed one year earlier: a case report. J Clin Pediatr Dent 1998;22(2):155-158.

7. Mourshed F. A roentgeographic study of dentigerous cysts: incidence in a population sample. Oral Surg Oral Med Oral Pathol 1964;18(1):47-53.

8. Benn A, Altini M. Dentigerous cysts of inflammatory origin: a clinicopathologic study. Oral Surg Oral Med Oral Pathol 1996;81(2):203-209.

9. Shear M. Cysts of the jaws: recent advances. J Oral Pathol 1985;14(1):43-59. 